\title{
Analysis of phosphate rock dissolution determining factors using principal component analysis in some acid Indonesian soils
}

\begin{abstract}
Dissolution of phosphate rock (PR) materials and its subsequent phosphorus (P) availability to plants depend upon soil characteristics, PR characteristics, type of crops, and environmental conditions. Agronomic effectiveness of the PR sources has frequently been investigated in the field or in the greenhouse. This is time consuming and not cost-effective. Therefore, identification of the soil characteristics influencing the dissolution of PR is very important for direct application of $\mathrm{P}$ sources. The principal component analysis was used to summarize the characteristics of acid soils in an incubation system into a number of factors that may affect PR dissolution. Three major factors were selected in this study: 1) soil texture, 2) soil acidity, and 3) fertilization. Using the scores of the individual factors as independent variables, a stepwise regression analysis was performed to derive a PR dissolution function. The coefficient of determination (R2) reached $0.91^{* *}$, and the magnitude of the different factors affect PR dissolution following the order of soil texture $(54 \%)>$ soil acidity $(43 \%)>$ fertilizer $(3 \%)$. Fertilizer was not significant as a PR dissolution factor.
\end{abstract}

Keyword: Acid soils; Dissolution-determining factor; Indonesia; Phosphate rock; Principal component analysis; Regression analysis 